\title{
Nucleosynthesis in the Hyades Open Cluster: Evidence for the Enhanced Depletion of ${ }^{12} \mathrm{C}$
}

\author{
Simon C. Schuler ${ }^{1}$, Jeremy R. King ${ }^{2}$, and Lih-Sin The ${ }^{2}$ \\ ${ }^{1}$ National Optical Astronomy Observatory, \\ 950 North Cherry Avenue, Tucson, AZ 85719, USA \\ email: sschuler@noao.edu \\ ${ }^{2}$ Dept. of Physics and Astronomy, Clemson University \\ 118 Kinard Laboratory, Clemson, SC 29634, USA \\ email: jking2@ces.clemson.edu, tlihsin@clemson.edu
}

\begin{abstract}
We present the results of a light element abundance analysis of three solar-type main sequence (MS) dwarfs and three red giant branch (RGB) clump stars in the Hyades open cluster using high-resolution and high signal-to-noise spectroscopy. The CNO abundances of each group (MS or RGB) are in excellent star-to-star agreement and confirm that the giants have undergone first dredge-up mixing. The observed abundances are compared to predictions of a standard stellar model based on the Clemson-American University of Beirut (CAUB) stellar evolution code. The model reproduces the observed evolution of the $\mathrm{N}$ and $\mathrm{O}$ abundances, as well as the previously derived ${ }^{12} \mathrm{C} /{ }^{13} \mathrm{C}$ ratio, but it fails to predict the observed level of ${ }^{12} \mathrm{C}$ depletion in the giants. More tellingly, the sum of the observed giant $\mathrm{CNO}$ abundances does not equal that of the dwarfs.
\end{abstract}

Keywords. nuclear reactions, nucleosynthesis, abundances- open clusters and associations: individual (Hyades), stars: abundances, stars: atmospheres, stars: evolution, stars: interiors

\section{Introduction}

The $\mathrm{CN}$ cycle is the dominant energy source powering the cores of stars more massive than the Sun. ${ }^{12} \mathrm{C}$ and ${ }^{14} \mathrm{~N}$ act as catalysts in the conversion of four ${ }^{1} \mathrm{H}$ nuclei into a single ${ }^{4} \mathrm{He}$ nucleus. In the presence of ${ }^{16} \mathrm{O}$, the $\mathrm{ON}$ cycle can also contribute to the energy production, and the two cycles working together make up the well-known CNO bi-cycle. There is no net loss of CNO nuclei in the CNO bi-cycle; however, the relative number of each nuclei changes due to different lifetimes to proton capture. The slowest reaction in the cycle, ${ }^{14} \mathrm{~N}(p, \gamma){ }^{15} \mathrm{O}$, creates a bottleneck and results in, relative to the initial abundances, a depletion of ${ }^{12} \mathrm{C}$, an enhancement of ${ }^{14} \mathrm{~N}$, and if the $\mathrm{ON}$ cycle is active, a depletion of ${ }^{16} \mathrm{O}$.

At the end of core H burning, stars experience the first dredge-up (Iben 1964), the expansion of the convective envelope from the surface layers down into the interior of the star. The first dredge-up mixes material processed by the $\mathrm{CN}$ and possibly the $\mathrm{ON}$ cycles up to the surface layers where the products of these core reactions can be observed. Stellar evolution models quantitatively predict changes in the surface abundances of red giant branch (RGB) stars that have experienced the first dredge-up. Here, we present the results of a CNO abundance analysis of three main sequence (MS) and three RGB clump giants in the Hyades open cluster. Using the dwarf abundances as a proxy for the initial composition of the giants, we compare the observed changes in the surface abundance of the giants to a standard stellar evolution model (Schuler et al. 2009). 


\section{Analysis}

We have used the Clemson-American University of Beirut (CAUB) stellar evolution code (The et al. 2007) to model the evolution of a $2.5 \mathrm{M}_{\odot}$ star, the approximate mass of the Hyades giants. The model is characterized by a metallicity $Z=0.025$ or $[\mathrm{Fe} / \mathrm{H}]$ $=+0.10$, the approximate metallicity of the Hyades cluster. The model was run through the core He burning phase, ending at about 785 Myr. The Hyades open cluster has an age estimated to be 600 Myr (Perryman et al. 1998), and the giants currently reside on the cluster RGB clump.

High-resolution, high signal-to-noise $(\mathrm{S} / \mathrm{N})$ spectra of the Hyades stars were obtained with the Harlan J. Smith 2.7-m telescope and "2dcoude" cross-dispersed echelle spectrometer at The McDonald Observatory. The spectra have a nominal resolution of $R=$ $\lambda / \Delta \lambda=60,000$ and $\mathrm{s} / \mathrm{n}$ ratios of $150-200$ for the dwarfs and $400-600$ for the giants.

The CNO abundances were derived assuming local thermodynamic equilibrium (LTE) using the LTE spectral line analysis and spectrum synthesis software package MOOG (Sneden 1973) along with model atmospheres interpolated from the grids of R.L. Kurucz. Stellar parameters are taken from Schuler et al. (2006). Carbon abundances were derived from $\mathrm{C}_{2}$ Swan lines at 5086.3 and $5135.6 \AA$, the $[\mathrm{C} \mathrm{I}$ ] forbidden line at $8727 \AA$, and for one of the giants, $10 \mathrm{CH}$ lines near $4325 \AA$. For N, three $\mathrm{CN}$ lines at $6706.7,6707.5$, and $6707.8 \AA$ were used, adopting the $\mathrm{C}$ abundances derived from the lines mentioned above. Oxygen abundances have been derived previously from the [O I] forbidden line at $6300 \AA$ by Schuler et al. (2006) using the same McDonald spectra discussed above. For each group (dwarfs or giants), the star-to-star abundances are in excellent agreement.

\section{Results \& Conclusions}

The observed ${ }^{14} \mathrm{~N}$ and ${ }^{16} \mathrm{O}$ abundances of the giants relative to those of the dwarfs are in excellent agreement with the model predictions: ${ }^{14} \mathrm{~N}$ is enhanced by a factor of 2.3 and no difference is seen in the ${ }^{16} \mathrm{O}$ abundances. The observations and model prediction are at odds, however, for ${ }^{12} \mathrm{C}$. The model predicts that the ${ }^{12} \mathrm{C}$ of the giants should be depleted by a factor of 1.5 ( $0.19 \mathrm{dex})$ relative to the dwarfs, but observationally, the giants have a ${ }^{12} \mathrm{C}$ abundance that is a factor of $2.33(0.37 \mathrm{dex})$ lower than the dwarfs. The 0.18 dex discrepancy represents a $\sim 6 \sigma$ result! Independent of the stellar model, the sum of the observed $C+N+O$ abundances for the giants is not equal to that of the dwarfs, as would be expected for material processed by the CNO bi-cycle. The abundance of the giants, $\log N(\mathrm{C}+\mathrm{N}+\mathrm{O})=8.91$, is only $79 \%$ of the dwarf abundance, $\log N(\mathrm{C}+\mathrm{N}+\mathrm{O})=9.01$. The model predicts that this sum should remain unchanged as a star evolves onto the RGB clump. Finally, the observed ${ }^{12} \mathrm{C} /{ }^{13} \mathrm{C}$ ratio of the giants has been previously determined by Tomkin et al. (1976), who found ${ }^{12} \mathrm{C} /{ }^{13} \mathrm{C}=21.0 \pm 1.8$, and Gilroy (1989), who found ${ }^{12} \mathrm{C} /{ }^{13} \mathrm{C}=25.8 \pm 1.4$. Both determinations are in good agreement with the model prediction, ${ }^{12} \mathrm{C} /{ }^{13} \mathrm{C}=23.4$ (with a MS value of $\sim 90$ ).

Random uncertainties in the mean abundances and systematic errors in the Hyades dwarf and giant parameter scales cannot account for the discrepancy in the observed and modeled ${ }^{12} \mathrm{C}$ abundances. Uncertainties related to our stellar evolution model have also been considered, but reasonable changes to the mass and metallicity of the model, as well as the adopted reaction rates, were unable to increase the destruction of ${ }^{12} \mathrm{C}$ enough to match the observations. Other mechanisms that could result in the preferential depletion of ${ }^{12} \mathrm{C}$, including the formation of C-rich grains in the atmospheres of the giants, are currently being investigated.

\section{References}

Iben, I. J. 1964, ApJ, 140, 1631

Perryman, M. A. C., et al. 1998, A\&A, 331, 81

Schuler, S. C., Hatzes, A. P., King, J. R., Kürster, M., \& The, L.-S. 2006, AJ, 131, 1057

Schuler, S. C., King, J. R., \& The, L.-S. 2009, ApJ, 701, 837

Sneden, C. 1973, ApJ, 184, 839

The, L.-S., El Eid, M. F., \& Meyer, B. S. 2007, ApJ, 655, 1058 